\title{
Controle de Congestionamento de Veículos Utilizando Sistemas de Transporte Inteligentes
}

\author{
Allan M. de Souza ${ }^{1}$, Leandro A. Villas ${ }^{1}$ (Orientador) \\ ${ }^{1}$ Instituto de Computação - UNICAMP \\ Avenida Albert Einstein, 1251 - 13.083-852 - Campinas-SP, Brasil \\ allanmselrc.ic.unicamp.br, leandrodic.unicamp.br
}

\begin{abstract}
Resumo. Mobilidade em grandes centros urbanos tornou-se um grande desafio devido aos congestionamentos de veículos recorrentes. Nesse contexto, Sistemas de Transporte Inteligentes emergiram para melhorar a eficiência do tráfego de veículos, porém, como detectar tais congestionamentos, principalmente na ocorrência de incidentes inesperados, e como controlá-los ainda são problemas em aberto. Este trabalho destina-se não apenas a evitar a iminência de congestionamentos mediante à incidentes inesperados, como também detectar, classificar e controlar congestionamentos constantes. Além disso, devido as características inerentes das redes veiculares, um novo protocolo para disseminação de alertas em redes veiculares foi proposto. As soluções propostas têm sido extensivamente comparadas com o estado da arte e os resultados mostram que elas são mais eficientes para reduzir os congestionamentos e melhorar a mobilidade em centros urbanos. Esta dissertação resultou em três artigos em periódicos e 8 trabalhos em conferências.
\end{abstract}

\section{Introdução}

Gargalos na infraestrutura de transporte, incidentes inesperados e o grande aumento no número de veículos devido ao crescimento populacional, geram diversos problemas de mobilidade nos grandes centros urbanos [de Souza et al. 2017]. Diante desse cenário, congestionamentos tornam-se um problema recorrente, não só provocando uma imprevisão do tempo de viagem, mas também gerando outros problemas, incluindo degradação da qualidade de vida, impactos negativos na economia regional e danos ambientais [de Souza et al. 2016a].

Os prejuízos causados pelos congestionamentos são evidentes. Só na cidade de São Paulo já ultrapassam a ordem de 50 bilhões por ano, onde, $85 \%$ estão relacionados ao tempo perdido, $13 \%$ ao consumo de combustível e $2 \%$ em emissões de gases poluentes, respectivamente [Cintra 2013]. Dessa forma, a fim de minimizar os congestionamentos e seus danos, Sistemas de Transporte Inteligentes (ITS - Intelligent Transportation Systems) emergiram [de Souza et al. 2017, Djahel et al. 2015]. Utilizando Redes Veiculares (VANETs - Vehicular Ad hoc Networks) como um de seus pilares, os ITSs permitem a integração e comunicação entre sensores, veículos e infraestruturas [de Souza et al. 2016a].

Por outro lado, Google Maps, TomTom e WAZE são algumas soluções existentes para minimizar os congestionamentos baseados em recomendações de rotas alternativas [Djahel et al. 2015]. Entretanto, essas soluções estão longe de atingirem os objetivos de um ITS e possuem limitações como: i) ausência de um mecanismo para planejamento de rota dinâmica, ii) não evitam o surgimento de congestionamentos causados por eventos inesperados e iii) as rotas sugeridas podem potencialmente transferir congestionamentos para outras áreas [de Souza et al. 2016a].

Para superar as limitações supracitadas, ITSs baseiam-se em informações reportadas periodicamente pelos veículos, incluindo velocidade média, posição e 
rota [Pan et al. 2016, Doolan and Muntean 2016]. Com essas informações, é possível construir um conhecimento sobre as condições de tráfego, e então, calcular rotas mais eficientes para os veículos. Porém, essas soluções não classificam os níveis de congestionamentos, assim, apenas empregam mecanismos para detecção de congestionamentos em intervalos pré-definidos. Sendo assim, essas soluções não detectam congestionamentos causados por eventos inesperados e também potencialmente detectam congestionamentos recorrentes muito tempo após sua ocorrência. Além disso, ITSs propostos em [Pan et al. 2012, Doolan and Muntean 2016] podem incorrer em roteamentos ineficientes, ou seja, podem potencialmente criar congestionamentos em outros locais. Por fim, é importante salientar que nenhum desses ITSs empregam mecanismos para tratarem os problemas relacionados as VANETs, como por exemplo, a sobre carga da rede devido a transmissões de mensagens redundantes ou múltiplas transmissões em um curto intervalo de tempo conhecidos como broadcast storm problem.

Soluções do estado da arte para controle de congestionamentos de veículos apresentam limitações, como troca de mensagens ineficiente, ausência de um mecanismo para controlar congestionamentos causados por eventos inesperados, intervalo de roteamento pré-definido e sugestões de rotas imprecisas [Bakhouya et al. 2011, Kim et al. 2008, Pan et al. 2012, Brennand et al. 2015, Doolan and Muntean 2016]. Com o intuito de minimizar o problema de broadcast storm em VANETs, diversas soluções foram propostas [Cunha et al. 2016]. Entretanto, nenhuma dessas soluções consideram o problema de sincronização introduzida pelo padrão IEEE 802.11p (descrito na Seção 2.2.2 em [de Souza 2016]), o qual é causado pela troca de canais empregada pelo padrão e degrada a performance do processo de disseminação de alertas em VANETs.

Para superar as limitações supracitadas, na dissertação, foram propostas soluções capazes de evitar a iminência de congestionamentos durante a ocorrência de incidentes inesperados, soluções para detectar, classificar e controlar congestionamentos recorrentes e um protocolo de disseminação de alertas ciente do problema de sincronização introduzido pelo padrão IEEE 802.11p utilizado nas VANETs. As soluções propostas avançam o estado da arte em detecção, classificação e controle de congestionamentos de veículos. Portanto, este trabalho tem potencial para melhorar a eficiência do tráfego de veículos em grandes centros urbanos, o que é uma necessidade nos dias de hoje. Em outras palavras, as soluções propostas apresentam uma melhor utilização da rede, maximizando a cobertura e reduzindo o número de transmissões, número de colisões e atraso. Além disso, melhoram a eficiência do tráfego de veículos reduzindo tempo de viagem, tempo de congestionamentos, consumo de combustível e emissão de gases poluentes.

\section{Principais Contribuições}

As principais contribuições da dissertação são as soluções propostas para: (i) disseminação de alertas em VANETs ciente do broadcast storm e sincronização introduzida pelo padrão IEEE 802.11 ; ; ii) controle de congestionamentos causados por incidentes inesperados; (iii) detecção, classificação e controle de congestionamentos recorrentes; e (iv) um algoritmo para o roteamento de veículos baseado em uma abordagem cooperativa.

Em resumo, podemos listar as seguintes contribuições:

1. Um protocolo para disseminação de alertas que trata os problemas broadcast storm e sincronização introduzida pelo padrão IEEE 802.11p.

Para minimizar o problema de broadcast storm, foi proposto um mecanismo baseado em atraso que prioriza veículos mais distantes para continuar o processo de 
disseminação. Além disso, foi proposto um mecanismo ciente da troca de canais para evitar a sincronização introduzida pelo padrão IEEE 802.11p. As Seções 4.3 e 4.5.1 em [de Souza 2016] apresentam a descrição detalhada do protocolo proposto e os resultados obtidos. Em resumo, os resultados mostram que o protocolo proposto apresenta uma cobertura maior que $90 \%$, reduz em até $68 \%$ a quantidade de mensagens transmitidas e reduz em $80 \%$ a colisão de mensagens, quando comparado com as soluções da literatura: Flooding, AID [Bakhouya et al. 2011], DBRS [Kim et al. 2008], DRIVE [Villas et al. 2014].

2. Uma solução pró-ativa distribuída para evitar a iminência de congestionamentos causados por incidentes inesperados.

A solução proposta, descrita em detalhes no Capitulo 4 de [de Souza 2016], é composta por três módulos: (i) detecção de eventos inesperados; (ii) disseminação de alerta; e (iii) gerenciamento do tráfego. Em linhas gerais, ao detectar um evento inesperado, como por exemplo acidentes de trânsito, a solução cria uma mensagem de alerta contendo informações do local do incidente e, aplicando o protocolo de disseminação de alertas (descrito no item 1), os veículos que se aproximam do local do incidente são alertados durante a fase de disseminação de alerta. Sendo assim, ao receber um alerta, os veículos são capazes de identificar se irão passar pelo local do incidente e calcular uma rota alternativa se necessário, assim, evitando o surgimento de congestionamentos no local. Os resultados mostram a eficiência da solução proposta, onde foi obtida um redução no tempo de viagem em pelo menos $32 \%$ e uma redução no tempo de congestionamento em pelo menos $88 \%$, quando comparada com as soluções da literatura DSP [Pan et al. 2012], RkSP [Pan et al. 2012] e With Routing [Brennand et al. 2015].

3. Uma solução eficiente para detecção, classificação e controle de congestionamentos recorrentes

A solução proposta, descrita em detalhes no Capitulo 5 em [de Souza 2016], utiliza um classificador baseado no algoritmo de K-Nearest Neighbors para detectar e classificar vias congestionadas [de Souza et al. 2016b]. Portanto, após detectar e classificar as vias congestionadas, a solução emprega um mecanismo eficiente para balanceamento de tráfego nas vias, consequentemente melhorando a eficiência do tráfego. Sendo assim, diferentemente das soluções da literatura, as quais detectam congestionamentos apenas em intervalos pré-definidos, a solução proposta permite detectar os congestionamentos assim que os mesmos ocorrem, permitindo controlá-los de forma mais efetiva. Os resultados mostram a eficiência da solução para detecção, classificação e controle de congestionamentos, onde foi possível reduzir o tempo de viagem e de congestionamento em até $24 \%$ e $67 \%$ respectivamente, quando comparada com as soluções da literatura DSP [Pan et al. 2012], RkSP [Pan et al. 2012] e With Routing [Brennand et al. 2015].

4. Um algoritmo para roteamento de veículos que utiliza uma abordagem cooperativa [de Souza et al. 2015]

$\mathrm{O}$ algoritmo cooperativo para roteamento de veículos, descrito em detalhes na Seção 5.4.2 em [de Souza 2016], visa distribuir melhor o tráfego de veículos. Assim, ao invés de calcular as melhores rotas para cada veículo (i.e., caminhos mínimos), o mesmo calcula rotas visando maximizar o fluxo de veículos na infraestrutura viária disponível. Portanto, alguns veículos podem per- 
correr distâncias maiores quando comparado com os caminhos mínimos, entretanto, as rotas sugeridas pelo algoritmo cooperativo contribui diretamente para um melhor fluxo de veículos, consequentemente reduzindo os congestionamentos. Os resultados mostram as vantagens da solução cooperativa, o algoritmo cooperativo proposto reduz o tempo de viagem e de congestionamentos em até $10 \%$ e $15 \%$ respectivamente, quando comparado com as soluções propostas em [Pan et al. 2012, Brennand et al. 2015, de Souza et al. 2016b].

Por fim, é importante salientar que a combinação de todas essas soluções deu origem ao ICARUS (Improvement of Traffic Condition through an Alerting and Re-routing System) [de Souza et al. 2016a], um sistema de gerenciamento de tráfego para melhorar a eficiência da infraestrutura de transporte. O ICARUS é capaz de detectar a origem dos congestionamentos e controlá-los devidamente de acordo com a origem. Em outras palavras, ICARUS é capaz de controlar efetivamente tanto congestionamentos causados por incidentes inesperados, quanto congestionamentos recorrentes. Os resultados mostram as vantagens de controlar congestionamentos de acordo com sua origem. Além disso, o ICARUS minimiza os problemas de comunicação presentes nas soluções existentes para disseminação de alertas em VANETs [Bakhouya et al. 2011, Kim et al. 2008, Villas et al. 2014], assim, maximizando a cobertura sem introduzir um atraso indesejado para o sistema, e também sem sobrecarregar a rede.

\section{Publicações}

Como resultado da dissertação de mestrado, tivemos diversas publicações: dois artigos em periódicos internacionais, um artigo em periódico latino-americano e sete artigos em conferências internacionais de prestígio e grande impacto na área de redes e sistemas distribuídos. Além disso, um artigo foram publicados no SBRC, o principal evento nacional na área de redes de computadores e sistemas distribuídos.

\section{Periódicos}

- 2016: Elsevier Computer Networks (Qualis A1, fator de impacto 1.9)

- 2016: International Journal of Distributed Sensor Networks (Qualis B1, fator de impacto 0.906)

- 2015: IEEE Transaction on Latin América (Qualis B4, fator de impacto 0.436)

\section{Conferências}

- 2016: IEEE Vehicular Technology Conference (Qualis A1, H-index 98)

- 2016: IEEE Symposium on Computers and Communication (Qualis A2, H-index 43)

- 2016: Simpósio Brasileiro de Redes de Computadores e Sistemas Distribuídos (Qualis B2, H-index 16)

- 2015: Dois artigos em International Conference on Computer and Information Technology (Qualis B1, Hindex 22)

- 2015: IFIP International Conference on New Technologies, Mobility and Security (Qualis B4, H-index 10)

- 2014: IEEE International Symposium on Network Computing and Applications (Qualis B1, H-index 31)

- 2014: ACM International Workshop on Mobility Management and Wireless Access (Qualis B3, H-index 15)

São necessários comentários sobre a qualidade dos veículos das publicações dos resultados obtidos na dissertação de mestrado, segundo sistema Qualis. A Tabela 1 mostra a porcentagem para cada tipo de publicação, de acordo com a classificação do atual sistema Qualis da CAPES.

Nota-se que $100 \%$ dos artigos publicados em periódicos internacionais foram publicados em periódicos classificados no extrato superior da CAPES (A1 e B1), considerados de primeira linha. Quanto aos artigos publicados em anais de conferência internacional, 72\% dos artigos publicados em anais de conferência internacional foram publicados 
Tabela 1. Porcentagem da produção científica classificada de acordo com o sistema Qualis

\begin{tabular}{|l|c|c|c|c|c|c|c|}
\hline & A1 & A2 & B1 & B2 & B3 & B4 & B5 \\
\hline Periódicos internacionais & 50 & - & 50 & - & - & - & - \\
\hline Periódicos latino-americanos & - & - & - & - & - & 100 & - \\
\hline Conferências internacionais & 14,28 & 14,28 & 42,88 & - & 14,28 & 14,28 & - \\
\hline Conferências nacionais & - & - & - & 100 & - & - & - \\
\hline
\end{tabular}

em conferências classificadas no extrato superior da CAPES (A1, A2 e B1), consideradas de primeira linha. Em nível nacional, os artigos foram publicados no Simpósio Brasileiro de Redes de Computadores e Sistemas Distribuídos (SBRC). Apesar de ser classificado como B2 no Qualis da CAPES, o SBRC é a principal conferência nacional da área de redes.

\section{Conclusão}

Este artigo resume as contribuições da dissertação de mestrado em [de Souza 2016]. O estado da arte em controle de congestionamento de veículos não consideram a origem dos congestionamentos, assim, controlando congestionamentos causados por eventos inesperados e congestionamentos recorrentes da mesma forma. Entretanto, identificar a origem dos congestionamentos contribui diretamente para maior efetividade em seu controle. Além disso, as soluções existentes não empregam soluções para tratar os problemas de comunicação inerentes as características das VANETs, consequentemente, essas soluções podem sobrecarregar a rede e introduzir um atraso indesejado. Por fim, embora existam soluções que tratam o problema de broadcast storm presente nas VANETs, nenhuma dessas soluções tratam a sincronização de transmissões introduzida pelo padrão IEEE 802.11p.

Para superar as limitações presentes no estado da arte, na dissertação, foram propostas soluções capazes de evitar a iminência de congestionamentos durante a ocorrência de incidentes inesperados, soluções para detectar, classificar e controlar congestionamentos recorrentes e um protocolo de disseminação de alertas ciente do problema de sincronização introduzido pelo padrão IEEE 802.11p utilizado nas VANETs. As soluções propostas avançam o estado da arte em detecção, classificação e controle de congestionamentos de veículos.

\section{Referências}

[Bakhouya et al. 2011] Bakhouya, M., Gaber, J., and Lorenz, P. (2011). An adaptive approach for information dissemination in vehicular ad hoc networks. J. Netw. Comput. Appl., 34(6):1971-1978.

[Brennand et al. 2015] Brennand, C. A. R. L., de Souza, A. M., Maia, G., Boukerche, A., Ramos, H., Loureiro, A. A. F., and Villas, L. A. (2015). An intelligent transportation system for detection and control of congested roads in urban centers. In 2015 IEEE Symposium on Computers and Communication (ISCC), pages 663-668.

[Cintra 2013] Cintra, M. (2013). A crise do trânsito em são paulo e seus custos. GVExecutivo, 12(2):58-61. 
[Cunha et al. 2016] Cunha, F., Villas, L., Boukerche, A., Maia, G., Viana, A., Mini, R. A., and Loureiro, A. A. (2016). Data communication in vanets: Protocols, applications and challenges. Ad Hoc Networks, 44:90 - 103.

[de Souza 2016] de Souza, A. M. (2016). Sistemas de Transporte Inteligente para Controle de Congestionamentos de Veículos. Universidade Estadual de Campinas - UNICAMP.

[de Souza et al. 2017] de Souza, A. M., Brennand, C. A., Yokoyama, R. S., Donato, E. A., Madeira, E. R., and Villas, L. A. (2017). Traffic management systems: A classification, review, challenges, and future perspectives. International Journal of Distributed Sensor Networks, 13(4).

[de Souza et al. 2016a] de Souza, A. M., Yokoyama, R., Boukerche, A., Maia, G., Cerqueira, E., Loureiro, A. A., and Villas, L. A. (2016a). Icarus: Improvement of traffic condition through an alerting and re-routing system. Computer Networks, 110:118 132.

[de Souza et al. 2015] de Souza, A. M., Yokoyama, R. S., Botega, L. C., Meneguette, R. I., and Villas, L. A. (2015). Scorpion: A solution using cooperative rerouting to prevent congestion and improve traffic condition. In 2015 IEEE International Conference on Computer and Information Technology (CIT), pages 497-503.

[de Souza et al. 2016b] de Souza, A. M., Yokoyama, R. S., Maia, G., Loureiro, A., and Villas, L. (2016b). Real-time path planning to prevent traffic jam through an intelligent transportation system. In 2016 IEEE Symposium on Computers and Communication (ISCC), pages 726-731.

[Djahel et al. 2015] Djahel, S., Doolan, R., Muntean, G.-M., and Murphy, J. (2015). A Communications-Oriented Perspective on Traffic Management Systems for Smart Cities: Challenges and Innovative Approaches. IEEE Communications Surveys Tutorials, 17(1):125-151.

[Doolan and Muntean 2016] Doolan, R. and Muntean, G. M. (2016). Ecotrec: A novel vanet-based approach to reducing vehicle emissions. IEEE Transactions on Intelligent Transportation Systems, PP(99):1-13.

[Kim et al. 2008] Kim, T.-H., Hong, W.-K., Kim, H.-C., and Lee, Y.-D. (2008). Information networking. towards ubiquitous networking and services. chapter An Effective Data Dissemination in Vehicular Ad-Hoc Network, pages 295-304. Springer-Verlag, Berlin, Heidelberg.

[Pan et al. 2012] Pan, J., Khan, M., Sandu Popa, I., Zeitouni, K., and Borcea, C. (2012). Proactive vehicle re-routing strategies for congestion avoidance. In Distributed Computing in Sensor Systems (DCOSS), 2012 IEEE 8th International Conference on, pages 265-272.

[Pan et al. 2016] Pan, J., Popa, I. S., and Borcea, C. (2016). Divert: A distributed vehicular traffic re-routing system for congestion avoidance. IEEE Transactions on Mobile Computing.

[Villas et al. 2014] Villas, L. A., Boukerche, A., Maia, G., Pazzi, R. W., and Loureiro, A. A. (2014). Drive: An efficient and robust data dissemination protocol for highway and urban vehicular ad hoc networks. Computer Networks. 